\title{
Tumores testiculares primarios en niños
}

\author{
Dra. Victoría Beresi R. 1,2; Dr. Humber to del Pozo P. 1,2; Dr. Juan Quintana B. 1,2 ; \\ Dr. Juan José Latorre L.2.3; Dr. César Jzzo S.2.4
}

Primary testicular tumors in children

\begin{abstract}
The cases of 37 children with testicular germ cell tumors attended at a metropolitan hospital of Santiago, Chile, between the years 1968 and 1984 are analized. The histologic types of their tumors were in 26 cases infantile embrional carcinoma (IEC), in 7 inmature teratoma and in 4 mature teratoma. The 26 IEC patients were treated with orchiectomy: $5 / 26$ with orchjectomy alone, 5/26 with orchiectomy phus lymphadenectomy (negative histology) and $16 / 26$ with orchiectomy plus chemoterapy using methotrexate, $D$ actinomycin and cyclosphosphamide (MAC). 2/7 patients with inmature teratoma were treated with orchiectomy alone, $2 / 7$ with orchiectomy plus lymphadenectomy and $3 / 7$ with orchiectomy and chemotherapy (MAC). 4/4 cases of mature teratoma were treated with surgery. Four of the 33 patients with malign germ cell tumors died, all of them with IEC: two were in stage II and they were treated with orchiectonly alone; the other two, instage I, were managed by orchiectomy and lymphadenectony, relapsed and died; $29 / 33$ patients are alive without evidence of disease af ter 1 to 16 years of follow up. Five IEC cases relapsed and 3 were treated with PVB (Cis-platinum, vinblastine and bleomicine) with good results. We think that malign germ cell tumors of the testes at stage I can be treated with surgery alone and must be followed by alphafetoprotein levels and chest radiograph. If they relapse ( $15 \%$ they can be treated and eured with chemo theraphy (PVB).
\end{abstract}

(Key words: Testicular tumors, prognosis, treatment, alphafetoprotein).

Los tumores testiculares (TT) representan el 1 a $2 \%$ de los tumores sólidos malignos 1 en la infancia y están en el séptimo lugar en orden de frecuencia. Su baja incidencia explica la razón por la que su tratamiento aún no esté bien definido. La mayoria de estos tumores (75 a $80 \%$ ) son de origen gesminal ${ }^{2}$ y entre estos predomina el carcinoma embrionario infantil (CEJ) o carcinoma del saco vitelino. Su diseminación se efectúa especialmente por vía hematógena ${ }^{2,3,4}$, a diferencia de los TT malignos del adulto que lo hacen por vía linfática, por lo que se justifica un mayor uso de quimioterapia sistémica y uno menor de linfadenectomía retroperintoneal y radioterapia.

Uno de los principales avances en los últimos años es el desarrollo de radioinmuno-análisis para los mareadores séricos: Alfafetoprote ína (AFP) y gonadotrofina coriónica humana (HGC.) que contribuyen al diagnóstico, clasificación y seguimiento de los tumores de células geminales ${ }^{5,6}$. La nás utilizada es la AFP que està ligada a la presencia del componente vitelino en el CEI.

1. Unidad de Oncología, Hospital Luis Catro Mackenna.

2. Departamento de Pediatría. Div. Ciencias Médicas Oriente, Facultad de Medicina, Universidad de Chile.

3. Servicio de Anatomia Patológica, Hospital Luis Calvo Mackenna.

4. Servicio de Cinugía, Hospital Luis Calvo Mackenna.
La HGC. tiene menos importancia ya que requiere la presencia del componente trofoblástico en los tejidos tumoralès y esto sucede en algunos teratomas inmaduros (TI) pero no en el CEI.

Las publicaciones sobre tumores testiculares de origen germinal en niños son escasas y describen sólo un pequeño número de pacientes atendidos a través de largos años $4,7,8$. Este trabajo no es la excepción, pero creemos que es interesante conocer la edad de presentación, tipos histológicos, estadio clínico inicial, tratamiento y sus resultados.

\section{MATERIALES Y METODOS}

Entre 1968 y 1984 ingresaron a la Unidad de Oncología del Hospital Luis Calvo Mackenna 802 niños con tumores sólidos malignos, de éstos, 44 tuvieron TT primarios $(5,4 \%) .19$ casos incluidos en este estudio fueron pubticados anteriormente. 9 La edad al diagnostico fluctuó entre 2 meses y 18 años (Figura 1). La clasificación histológica de estos tumores se hizo de acuerdo al criterio de la OMS.10 37 de los 44 niños (84\%) tuvieron tumores de células germinales: $26 \mathrm{CEJ}$, 7 Teratomas Malignos y 4 Teratomas Benignos. Los 7 restantes tuvieton Rabdomiosarcoma que analizaremos en una publicación posterior.

Todos se estudiaron con radiografía de tórax al ingreso. En algunos pielografía de eliminación y cintigrafía ósea. Linfografía pedia se hizo excepcionalmente por dificultades técnicas derivadas de su corta edad.

La alfafetoproteina (AFP) determinada por radio- 
inmuno-análisis, se efectuó en los $\mathbf{1 2}$ últimos pacjentes. En 8 se réalizó entre 1 día antes de la operación y 21 días después de ella (mediana 12 días). En 4 pacientes se efectuó sólo después de 30 días de la cirugía.

En relación a la extensión de la enfermedad al điagróstico se usó la clasificación de Mostofí y Price 11 : 34/37 tuvieron estadio l (localizado al testículo) 3 Estadio II (metastásis en abdomen), ninguno Estadio [1I (metástasis sobre diafragms o metástasis a distancia).

Tratamiento: en todos los pacientes efectuó orquiectomía radical por vía inguinal. La linfadenectomia retroperitonéal uni]atcral sólo se realizó en los 7 primeros pacientes. Además se emplearon dos tıpos de qui: mioteraia múltiple:

MAC (metotrexato: $20 \mathrm{mg} x \mathrm{~m} 2$ i.v, actinomicina D: $0,4 \mathrm{mg} \times \mathrm{m} 2$ i.y. $y$ ciclofosfamida: $400 \mathrm{mg} \times \mathrm{m} 2$ i.v. semanal durante 3 meses y luego cada 15 diasi. Recibieron este tratamierto 19 pacientes por períodos que variaron entre 2 y 18 meses.

PVB (cisplatinum: $100 \mathrm{mg} \times \mathrm{m} 2$ i.y. dia 1, vinblastina: $3 \mathrm{mg} x \mathrm{~m} 2 \mathrm{i}$. $\mathrm{m} 2$ 1.v. día 1 y luego cada 7 días por 3 veces. Estos ciclos se repiticron cadd 21 días por 3 veces. Esta modalidad se usó en 3 pacientes que recayeron.

Los pacientes con tumores malignos se controłaron entre 3 y 16 años excepto el últino que leva un año do control y se consideran curados los que tienen una sobre. vida libre de enfermedad de 2 anos ya que las recaidas después de este periodo son excepcionales.

El análisis estadístico se ofectuó a través de las tablas de sobrevida de Kaplán y Meier ${ }^{25}$.

\section{RESULTADOS}

Se analizará cada tipo histológico por separado. Los resultados obtenidos con las diferentes modalidades de tratamiento se muestran en la Figura 2.

Carcinoma embrionario infantil. De 37 tumores germinales 26 fueron clasificados como CEI. La edad al ingreso fluctuó entre 9 y 51 meses (mediana 18 meses) (Fig. 1). 24 pacientes tuvicron Estadio Clínico I y 2 Estadio II.
Se utilizaron 5 modalidades de tratamiento: Con orquiectomia exciusiva: se trataron 4 pacientes, 2 correspondieron a Estadio Clínico II y fallecieron con metástasis abdominales extensas 19 y 21 días después de operados. Los otros 2 , Estadio I, viven más de 10 arros sin evidencia de enfermedad (see).

Con arquiectomia y linfadenectomia retroperitoneal unilateral: En los 5 pacientes el estudio histológico de los ganglios abdominales fue negativo. Dos fallecieron 23 y 25 meses después de la orquiectomía: en el primero se encontraron metástasis pulmonares a los 18 meses del diagróstico, falleció 5 meses después y en su necropsia se encontraron, además, múltiples metástásis en ganglios lumboaórticos y mediastínicos; el segundo, de 10 meses de edad, tenía síndrome de Down, sufrió 2 recaídas inguinales y una tercera inguinal y lumboaórtica, falleció con metástaśis pulmonares 25 meses después de la orquiectomía y no se le hizo autopsia.

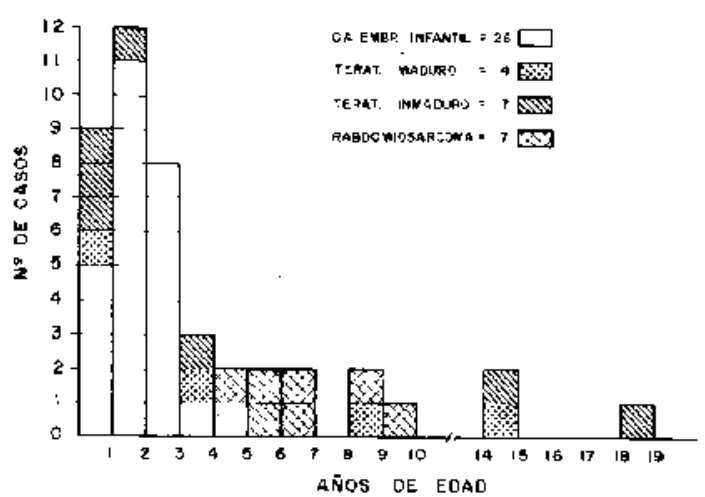

Figura 1. Distribución por edad en el momento del diagnóstico de 44 tumores testiculares primarios en nin̄os.

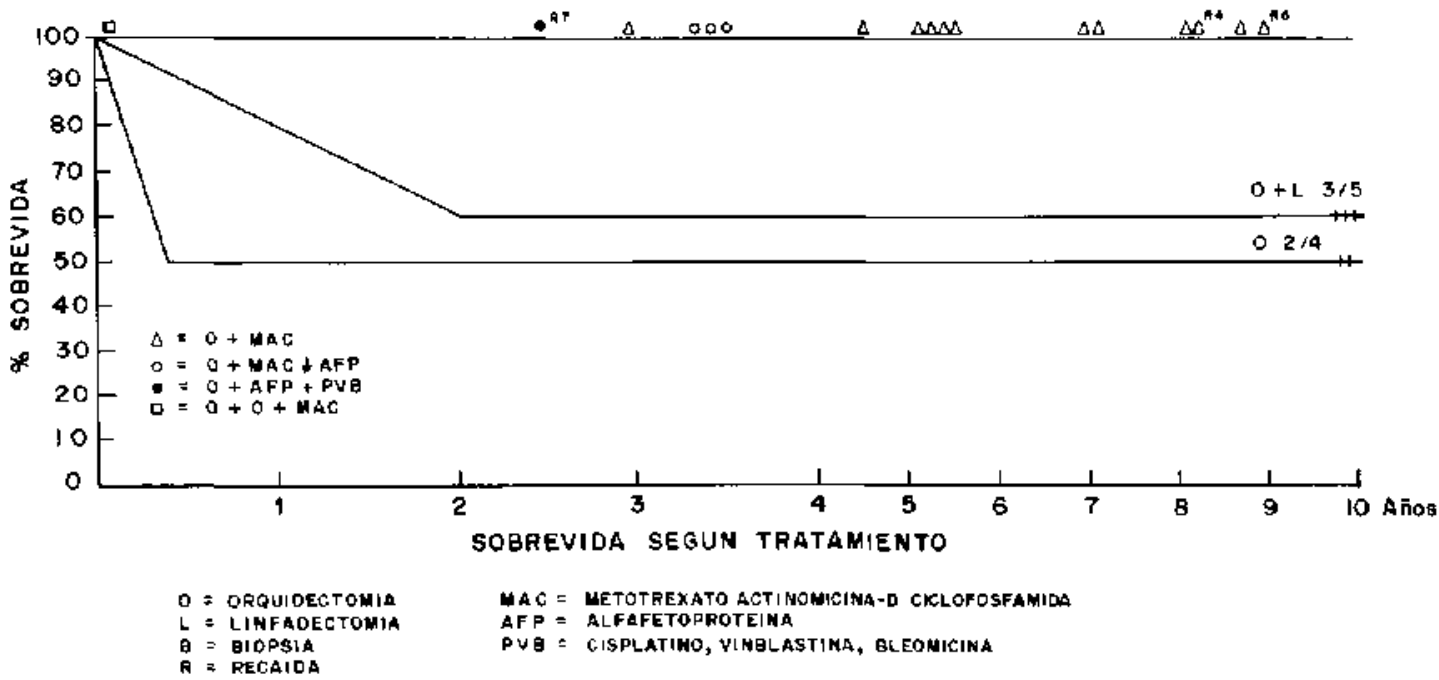

Figara 2. Resultados del tratamiento en 26 nin̄os con carcinoma embrionario testicular. 
Con orquiectomia y quimioterapia (MAC. 12 a 18 meses ): Después de la orquiectomia, 12 nifios recibieron quimioterapia MAC durante 12 a 18 meses. Todos estaban vivos, sin evidencia de enfermedad, entre 14 y 112 meses (mediana 80 meses). Dos pacientes recayeron: el primero en región inguinal a los 7 meses, se operó y se agregó vinctistind al $\mathrm{MAC}(1,5 \mathrm{mg} \times \mathrm{m} 2$ durante 18 meses). Actualmente está en remisión completa 308 meses después del diagnóstico. El segundo paciente, con recaída testicular, fue referido a nosotros con el antecedente de biopsia testicular 2 dias antes de la orquiectomía: la AFP fue positiva $>5.000 \mathrm{ng} \times \mathrm{ml}$; a los 3 meses de iniciada la quimioterapia tuvo la primera recaída local que se extispó quirúrgicamente en torma aparentemente total, sin embargo, 3 meses más tarde aparecieron metástasis inguinales y abdo. minales que se extirparon parcialmente. Se cam'bió la quimioterapia a vinblastina $1,4 \mathrm{mg}$ i.v. cada 6 horas durante 2 días y bleomicina $30 \mathrm{mg}$ i.v. diarios durante 5 dias (VB). Luego del primer ciclo, se obtuvo remisión parcial del tumor por lo que recibió radioterapia abdominal con Co60. $45 \mathrm{~Gy}$. Después del segundo ciclo, se logró remisión completa y la AFP se normalizó (7 ng $x$ mi). Ambos ciclos de vinblastina y bleomicina fueron seguidos por neutropenia severa, complicada con sepsis, una causada por germen desconocido y otra por Escherichia coli. Los padres rehusaron continuar el tratamiento, sin embargo e] niño está bien 97 meses después de la segunda recaída.

Orquiectomia y quimioterapia (MAC $2 \cdots 7$ mescs): con AFP normal 30 días después de cirugía: este grupo está formado por 4 nin̄os que tuvieron valores iniciales de AFP entre 110 y $370 \mathrm{ng} \times \mathrm{ml}$. Dos se trataron con quimioterapia 7 meses y dos durante 2 meses. Los 4 están bien entre 41 y 54 meses (mediana 49 meses).

Con Cinigia sola y AFP también nomalizada antes del mes de operado: es un solo caso que llegó al setvicio después de orquiectomia: se normalizó AFP a los 30 días $(8 \mathrm{ng} \times \mathrm{ml}$ ). Se deciđió controlarlo mensualmente con AFP y xadiografías de tórax. Siete meses y medio después de la orquiectomía el AFP subió a $>400 \mathrm{ng}$ $x$ ml. Et niño presentó una recaida en ganglios linfáticos lumboaórticos que produjeron exclusión renal izquierda, demostrada por pielografía descendente. Se trató con quimioterapia PVB y en un mes se obtuvo remisión completa que se mantiene 29 meses después de esta recuída.

En resumen, de estos 26 pacientes, fallecieror $4(15 \%)$ ( 2 en Estadio II inicial y 2 con Estadio I inicial, con metástasis pulmonares a pesar de linfadenectomía negativa): ninguno se trató con
QT (1968-1972). Otros 3 niños recayeron a los 6,7 y $71 / 2$ meses después de la orquiectomía respectivamente: se trataron con diferentes esquemas de quimioterapia (VB y PVB) y están sin evidencia de enfermedad 108,97 y 29 meses después del diagnóstico.

Teratoma benigno (TB). Hemos atendido 4 niños con este diagnóstico cuyas edades variaron entre 11 meses y 14 años (Fig. 1). Se trataron con orquiectomía por vía inguinal y ligadura alta del cordón. La AFP se realizó en uno y fue normal (12 $\mathrm{ng} x \mathrm{ml}$ ). Los 4 están bien entre 15 meses y más de 10 años de seguimiento.

Teratoma maligno (TM). Se trataron 7 pacientes, entre 2 meses y 18 años (mediana 14 meses). En todos se practicó orquiectomía. En 2 fue el único trataniento, en otros 2 se hizo linfadenectomía unilateral (la histología dio resultados normales en los linfáticos extrajdos) y en 3 pacientes se agregó quimioterapia (MAC). EI único enfermo Estadio II fue un adolescente de I8 años cuyo estudio histológico demostró teratoma inmaduro con elementos de carcinoma embrionario, coriocarcinotha y seminoma. La linfografía pedial resultó normal, sin embargo la AFP, que fue de $210 \mathrm{ng} x \mathrm{ml}$ antes de la orquiectomía, se elevó $>\mathbf{4 0 0} \mathrm{ng} \times \mathrm{ml}$. Se realizó laparotomia exploradora que demostró metástasis en los gnaglios linfáticos, comprobadas con estudio histológico. Se trató con MAC semanal, pero después de $S$ dosis su enfermedad prosiguió, la AFP continuó positiva y la gonadotrofina coriónica humana (HCG) ${ }^{13}$ fue de $66 \mathrm{mU} \times \mathrm{ml}$ (normal $>2 \mathrm{mU} \times \mathrm{ml}$ ). La quimioterapia se cambió por PVB. Después de 2 cíclos se obtuvo remisión completa con normalización de AFP y HCG. Se efectuaron 4 ciclos cada 21 días y el enfermo continúa bien, con marcadores séricos normales, 36 meses despucs del diagnóstico. Todos los pacientes con teratoma maligno están vivos entre 3 y 16 años (mediana 12 años).

\section{DISCUSION}

E] pronóstico de los TT primarios malignos ha mejorado con los actuales tratamiento de cirugía, radioterapia y quimioterapia múltiple $13,15,19$ Sir embargo, el uso combinado de estos métodos de tratamiento es un tema de controversia, especialmente en el Estadio I. El tratamien to fundamental es la orquiectomía radical por vía ingu inal y con ella sola se obtuvo un 50 a $60 \%$ de curación en el Estadio I $^{2.13}$. En nuestra serie se realizó este tratamiento exclusivo en 6 pacientes ( $4 \mathrm{CEI}$ y $2 \mathrm{TI}$ ) y los 4 niffos con Estadio I están vivos más de I0 años. Los 2 que fallecieron (CEI) fueron Estadios II iniciales y hoy se sabe que 
estos pacientes deben tratarse además con quimioterapia múltiple.

Actualmente la sobrevida libre de enfermedad ha aumentado a $85 \%$ y la sobrevida total a $100 \%$ gracias al control estricto de los pacientes con AFP $y$ radiografías de torax ${ }^{15}$, lo que permite diagnosticar precozmente las recaídas (15\%) y hacer su tratamiento de inmediato. En nuestra casuística, tratamos 2 niños, con quimioterapia durante 2 meses y uno sin ella, por normalización de la AFP: este último recayó 7 meses después de orquiectonía, se trató luego con PVB y contínúa 29 meses sin evidencia de enfermedad. Creemos que la quimioterapia tan breve en los dos primeros enfermos no influyó en este resultado.

El uso de la linfadenectomía retroperitoneal es motivo de discusiones $2,3,4$ debido a que el estudio histológico de los ganglios extirpados es en general negativo $y$, sin embargo, algunos de estos pacientes fallecen por metástasis pulmona. res $^{24}$ : asi ocurriô en 2 de 7 pacientes en que practicamos este procedimiento, por lo que en 1975 dejamos de usardo

La quimioterapia profiláctica en pacientes con Estadio 1 ha demostrado ser útil ya que todos los pacientes tratados han curado 2.8 .9 : $\operatorname{los} 12 \mathrm{pa}$ cientes tratados con $\mathrm{MAC}$ en esta serie están vivos con más de 40 meses de seguimiento, d pesar de que 2 recayeron. Sin embargo, creemos que este tratamiento no se justifica en todos los pacientes dado el resultado que se obtiene con cirugía sola y control posterior mensual con AFP y radiografía de tórax. La quimioterapiń puede ser útil en los enfermos con Estadio I en los cuales la detección precoz de las metastasis no pueda efectuarse por no disponer de estos exámenes.

La quimioterapia combinada ha demostrado ser realmente útil en los estadios avanzados: $\mathrm{cos}^{\circ}$ ella se obtiene remisión completa aproximadamente en $58 \%$ de los enfermos, porcentaje que aumenta en $30 \%$ cuando se puede realizar extirpación quirúrgica completa del tumor residua 19,21 . Si la extirpación es incompleta puede usarse radioterapia externa, única indicación de este tratamiento en estos casos.

Las combinaciones đe medicamentos que han demostrado ser útiles en los TT germinales malig. nos son: vincristina, actinomicina $D$ y ciclofos. famida (VAC), metotrexato, actinomicina D y ciclofosfamida $(\mathrm{MAC})^{15}$ y la combinación de vinblastina, bleomicina y cisplatinum (PVB) ${ }^{17,18}$. Este último tratamiento se usa especialmente en Estadios 11 y III. Su toxicidad, sin embargo, es mayor que VAC o MAC. Debido a ello, últimamente se ha reemplazado la vinblas. tina por etoposida (VP 16): con ésta se obtienen resultados terapéuticos similares, pero con mejor tolerancia ${ }^{20}$. Las altas dosis de cisplatinun tienen serios riesgos de nefrotoxicidad y escasa aceptación de los pacientes debido a naúseas y vómitos intensos. Por esta razón el uso de esta droga en infusión continua durante 5 días, con igual dosis total (100 $\mathrm{mg} \times \mathrm{m}^{2}$ i.v.), tiene el mismo efecto; pero menor toxicidad ${ }^{2} \mathrm{I}$.

La duración de estos tratamientos ha sido variable, algunos autores la usaron 24 meses, 12 meses $^{15}$ y por último se ha establecido que es útil un tratamiento de inducción con 4 ciclos de PVB sin tratamiento de mantención ya que los resultados son similares 22,23 .

\section{RESUMEN}

Se analizan 37 niños con tumores testiculares de células germinales atendidos en el Hospital Luis Calvo Mackenna entre 1968-1984. Los tipos histológicos fucron 26 carcinomas embrionarios infantiles (C.E.l.) 7 teratomas inmaduros y 4 teratomas maduros. Los 26 carcinomas embrionarios se trataron con orquiectomia total, 5 con orquiectomía exclusiva, 5 con orquiectomía y linfadenectomía (histología negativa) y 16 con orquiectomia y quimioterapia M.A.C. (metotrexato, actinomicina D y ciclofosfamida). Los últimos 12 pacientes ingresados se controlaron con alfafetoproteina. De los 7 teratomas inmaduros la orquiectomía fue exclusiva en 2 , en 2 se agregó linfadenectomia y en 3 quimioterapia (M.A.C.). En los 4 teratomas maduros se utilizó orquiectomía exclusiva. Fallecieron 4/33 pacientes con tumorcs malignos de células germinales, todos CEI, de estos 2 exan Estadio II y se trataron con orquiectomía exclusiva. 2 Estadio I tratados con orquiectomía y linfadenectomía recayeron y fallecieron. Los 29 restantes están vivos sin evidencia de enfermedad entre a y 16 años, a pesar de que recayeron $5 \mathrm{CEI}, 3$ de los cuales se trataron con PVB (cis-platinum, vinblastina y bleomicina) con buen resultado. Los tumores de células germinales testiculares malignos, en Estadio I pueden ser tratados solo con cirugía y controlados con niveles de alfafetaproteina y radiografía de tórax. Si recidivan (menos del $15 \%$ ), pueden ser curados con quimioterapia (PBV).

\section{REFERENCIAS}

1. Young, J.L., JP., Miller, R.W.: Incidence of malignant tumors in U.S, children. J Pediatr 86: 254 , 1975.

2. Brosman, S.A.: Testicular tumors in propubertal children Urology 13: 581, 1979.

3. Bracken, R.B., Johnson, D.E., Cangir, A. and Ayala, $A$ : Regional lymph nodes in infants with embrional carcinoma of testis. Urology 11: 376, 1978. 
4. Ise. T, Ohtsuki, M., Matsumoto, Kand Sauo, R.: Management of malignant testicular tumors in children. Cancer 37: 1539, 1976.

5. Tsuchide, Y., Saito, S., Ishida, M., Ohmi, $K$., Urano, Y., Endo, Y. and Oda, T.: Yolk sac tumor (endodermal sinus tumor and alpha-fetoprotein). Cancer 32: 917,1973 .

6. Chuitz, H., Sell. A., Norgaard-Pedersen, B. and Arends, J.: Serum alpha-fetoptotein and human chorionic gonadotropin as markers for the effect of postoperative radiation therapy and for chemotherapy in testicular cancer. Cancer 42: $2182,1978$.

7. Karamehmedovic, O., Woodti, W. and Pluss, M.J.: Testicular tumor in childhood. I Ped Surg 10: 109. 1975.

8. Sabio. M. Burget, E.O., Jr., Farrow, G.M. and Kelolis, $P_{\text {.: }}$ Embryonal carcinoma of the testis in childhoud. Cancer 34: $2118,1974$.

9. Quintana, J., Beresi, V., Latorre, J.J., Izzo, C., Sanhueza. S. and Del Pozo, $H$.: Infantil embryonal carcinoma of the testis. J Urol 128: 785, 1981.

10. C.I.E.O.: Clasificación internacional de enfermedades para oncologia, $1^{\circ}$ sd. O.P.S. - O.M.S., Washington, D.C., 1977 .

11. Mostofi, F.K., Sóbin, L.H. International Classification of tumors of the testis (No 16) Geneva: W.H.O., 1976.

12. Kaplan, W.E. Arenson, E., Cromie, W., Homsy, $Y_{\text {, }}$ Demers, J.: Yolk Sac Tumors. Dialogues in Pediat ic Lrology, 5: 3, 1982.

13. Hopkins, T.B., Jaffe, N., Colodny. A., Cassedy, J.R. and Filler, R.M.: The management of testicular tumors in children. J Lrol 120: 96, 1978.

14. Exelby, P.R.: Testicular cancer in clilitren, Cáncer, 45: $1803,1980$.

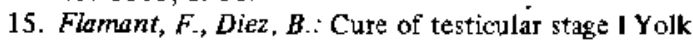
sac tumor (endodermal sinus tumor) in children by conserva tive treatment. Prac Am Soc Clin Oncol 4 : 235,1985 .
16. Brodeur, G.M., Howarth, C.B., Pratt, C.B. Cares, J. and Muster. O.: Mabignant germ cell tumors in 57 children and adolesecents. Cancer 48: 1890, 1981 .

17. Williams C.: Current dilemmas in the management of ronseminomatous germen cell tumors of the testis. Cancer Treat Rev 4: 275, 1977.

18. Sinhom, L.W., Donohue, J.P.: Cis-diamminodichloroplatinum, vinblastina, and bicomicina combination chemotherapy in disseminated testicular cancer. Am Intern Med 87: 293, 1977.

19. Pizzocaro, G., Salviani, R., Pasi, M. Zamoni, $F$. . Milani, $A$, Filotti, $S$ and Monfardini, S. Carly resection of residual tumor during Cisplatinum, Vinblastina, Bleomycin combination chemotherapy in stage IIl and bulky stage II non-seminomatous testicular cancer. Cancer 56: 249, 1985.

20. William, S., Einhorn, L, Grecas, A., Birch, R. and Irwin. I.: Disseminated Germ Cell Tumors. A comparision of Cisplatinum plus Bleomycin plus Vinblastine (P.V.B.) or V.P. 16 (B.E.P.) Proc Am Soc Clin Oncol 4: 99, 1985.

21. Richarson, R.L., Wahn, R, K1ols, L.K., Long. H. et als.: Bleomy cinc (B), Etofoside (F) and continous infusion Cisplatinum (P) (B.E.C.I.P.) in metastasis testicular encer. Proc Am Soc Clin Oncol 4: 105, 1985.

22. Wirgin, D., Whitémore, W.F., Golbey, R.B. V.A.B. ${ }^{6}$ combination chemotherapy without maintenance in treatment of disseminated cancer of the testis. Proc Am Soe Clin Oncol 4: 109, 1985.

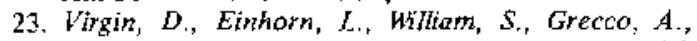
Birds, $R .:$ A multi-institutional experience with P.V.B. H/A, with no mainienance in advanced germ cell tumors of the tcstis. Proc Am Soc Clin Oncol 4: 109, 1985.

24. Young, P.G., Mount, B.M., Foote, F.W., Jr. and Whitemore, W.F. It.: Embrional adenocarcinoma with the pubertal testis. Cancer 26: $1065,1970$.

25. Meir, $P .:$ Stadistic and Medical Experimentation. Bionetrics $31: 511,1975$. 